See Article page 89.

\section{Commentary: Patience is a virtue}

\author{
Christoph Haller, MD
}

Mechanical assist devices are increasingly establishing their role in the treatment of pediatric heart failure. Since the inception of the Pediatric Interagency Registry for Mechanical Circulatory Support (PEDIMACS), more than 500 durable and temporary devices have been implanted, with a $74 \%$ survival at 6 months according to this registry. ${ }^{1}$ However, mechanical support for these patients faces unique challenges, driven by the heterogeneity of the pediatric population, off-label use of devices approved for adults, and limited economic appeal for the development of pediatricspecific devices. Patients age $<1$ year are at particularly high risk and have the highest mortality while on support. Recovery on mechanical support is particularly rare in both children and adults. ${ }^{1,2}$ Thus, it is all the more encouraging to see reports of successful assist device weaning in the very young, which can potentially help identify both optimal therapeutic strategies and the patients that will benefit the most.

In this issue of the Journal, Philip and colleagues ${ }^{3}$ present 2 cases of relatively late ventricular functional recovery on biventricular paracorporeal pulsatile assist devices in patients age $<1$ year. ${ }^{3}$ Both patients showed gradual improvement in left ventricular function and indexed left ventricular end-diastolic volume and were subsequently evaluated for ventricular assist device (VAD) weaning with a structured weaning trial over the course of 5 days. Before weaning, invasive hemodynamic data were acquired by catheterization to further objectify adequacy of ventricular function. Follow-up confirmed sustained recovery at 18 and 21 months after VAD removal.

\footnotetext{
From the Department of Cardiovascular Surgery, The Labatt Family Heart Centre, The Hospital for Sick Children, University of Toronto, Toronto, Ontario, Canada. Disclosures: The author reported no conflicts of interest.

The Journal policy requires editors and reviewers to disclose conflicts of interest and to decline handling or reviewing manuscripts for which they may have a conflict of interest. The editors and reviewers of this article have no conflicts of interest.

Received for publication April 21, 2020; revisions received April 21, 2020; accepted for publication April 22, 2020; available ahead of print May 11, 2020.

Address for reprints: Christoph Haller, MD, Division of Cardiovascular Surgery, The Hospital for Sick Children, 555 University Ave, Toronto, ON, Canada, M5G 1X8 (E-mail: christoph.haller@sickkids.ca).

JTCVS Techniques 2021;5:95-6

2666-2507

Copyright (C) 2020 The Authors. Published by Elsevier Inc. on behalf of The American Association for Thoracic Surgery. This is an open access article under the CC BY-NCND license (http://creativecommons.org/licenses/by-nc-nd/4.0/).

https://doi.org/10.1016/j.xjtc.2020.04.027
}

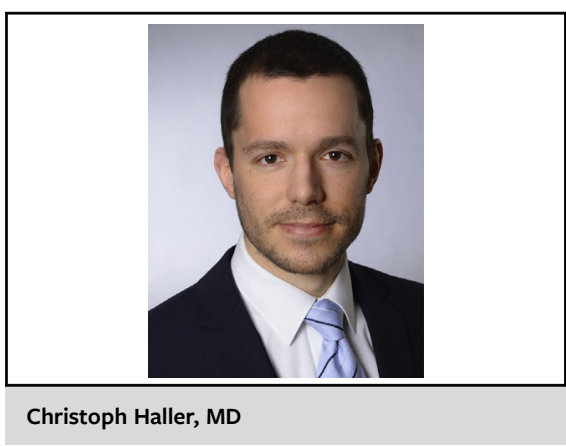

CENTRAL MESSAGE

Myocardial recovery can occur

late during mechanical support

despite an unfavorable etiology

of ventricular dysfunction.

Recovery of ventricular function on durable mechanical support is not unheard of in the pediatric population. $\mathrm{Pa}$ tients on durable assist devices have lower recovery rates compared with patients on temporary devices, reflecting the differing indications for support. ${ }^{1}$ Recovery rates also depend on age and underlying diagnosis, with patients age $<2$ years and those with nonchronic conditions, such as myocarditis, have a greater likelihood of successful assist device weaning ${ }^{4}$; however, the 2 cases reported by Philip and colleagues have characteristics that make functional recovery less likely.

Successful long-term VAD support aimed at recovery and subsequent explantation has been shown in and advocated for the pediatric population. ${ }^{5}$ Although Philip and colleagues indicate that recovery is commonly seen early after assist device implantation, types of myocardial responses to ventricular unloading must be taken into consideration. Early recovery is commonly seen in patients with nonchronic etiologies, such as postoperative stunning, myocarditis, and temporary ischemic insult. However, longer-term unloading strategies have shown structural remodeling with reduced fibrosis and increased cardiomyocyte numbers even in patients with chronic etiologies, such as dilative cardiomyopathy. ${ }^{6}$ Whether myocardial recovery is improved on pulsatile support compared with continuous-flow devices remains unclear, ${ }^{6,7}$ but it is well established that a subgroup of patients may qualify for a bridge-to-recovery strategy. Unfortunately, we lack clear identifiers of patients likely to recover on long-term mechanical support. More granular data are needed on the likelihood of sustained recovery to guide clinical decision making. 
Until then, clinicians have to stay vigilant, closely following patients on mechanical assist devices throughout their time on support. VAD programs must ensure that proper protocols are in place not only to assess patients with signs of myocardial recovery for potential weaning, but also to optimize support strategies to improve recovery itself. Diligent analysis and reporting of our experiences are essential. The most important conclusion to be drawn from the report by Philip and colleagues is that recovery can occur late even in patients on a long-term strategy not primarily aimed at recovery. Patience is the virtue needed both to gain a better understanding of the pathophysiology of myocardial recovery and to identify weaning candidates on long-term mechanical support.

\section{References}

1. Morales DLS, Rossano JW, VanderPluym C, Lorts A, Cantor R, St Louis JD, et al Third annual pediatric interagency registry for mechanical circulatory support (pedimacs) report: preimplant characteristics and outcomes. Ann Thorac Surg. 2019;107:993-1004.

2. Teuteberg JJ, Cleveland JC Jr, Cowger J, Higgins RS, Goldstein DJ, Keebler M, et al. The Society of Thoracic Surgeons intermacs 2019 annual report: the changing landscape of devices and indications. Ann Thorac Surg. 2020;109: 649-60.

3. Philip J, Machado D, Shih R, Shenoy A, Ebraheem M, Sullivan K, et al. Myocardial recovery following pulsatile biventricular assist device support in infants: report of 2 cases. J Thorac Cardiovasc Surg Tech. 2021;5:89-92.

4. Miera O, Germann M, Cho MY, Photiadis J, Delmo Walter EM, Hetzer R, et al. Bridge to recovery in children on ventricular assist devices-protocol, predictors of recovery, and long-term follow-up. J Heart Lung Transplant. 2018;37: 1459-66.

5. Adachi I, Burki S, Fraser CD Jr. Current status of pediatric ventricular assist device support. Semin Thorac Cardiovasc Surg Pediatr Card Surg Annu. 2017;20:2-8

6. Kasten J, Rakheja D, Zhang S, Thankavel P, Das BB. Reverse histologic remodeling after mechanical unloading of failing hearts in children with dilated cardiomyopathy. J Heart Lung Transplant. 2017;36:1268-71.

7. Krabatsch T, Schweiger M, Dandel M, Stepanenko A, Drews T, Potapovkjl E, et al. Is bridge to recovery more likely with pulsatile left ventricular assist devices than with nonpulsatile flow systems? Ann Thorac Surg. 2011;91: $1335-40$. 